\title{
A Southern Sky Survey of Low Mass Stars and Brown Dwarfs
}

\author{
Andrew D. Thackrah \\ Institute for astronomy, University of Edinburgh, Edinburgh, Scotland \\ Michael R. S. Hawkins \\ Royal Observatory, Edinburgh, Scotland
}

\begin{abstract}
We are conducting a survey of the southern sky to study the properties of the lowest mass stars and the sub-stellar brown dwarf population thought to exist at masses below $0.08 M_{\odot}$. The survey makes use of plates from the UK and ESO Schmidt telescopes, using the new SuperCOSMOS plate measuring machine at Edinburgh to provide a catalogue of photographic BRI colours and proper motion measurements. Candidates are selected by extreme R-I colour $(>2.5)$ to a plate-limited magnitude of $I \approx 19.0$ and $R \approx 21.0$. The size of the survey is only limited by the progress of the UK Schmidt I band survey and the availability of photometric data for calibration of SuperCOSMOS measurements. It is anticipated that the survey will form part of a multi-colour proper motion database of southern sky objects.
\end{abstract}

\section{The extent of the survey}

The UK Schmidt's $B_{J}$ survey using the IIIaJ emulsion and GG395 filter combination is now complete as is the ESO Schmidt $R$ band IIIaF/RG630 survey. The UK Schmidt is also conducting I (IV-N/RG715) and 'OR' (IIIaF/OG590) surveys which when complete will provide a photographic BRI record of the southern sky. This project makes use of many of the survey plates, with supplementary non-survey plates of similar grade where neccessary. Our survey fields have been chosen according to photometric and astrometric considerations discussed in sections 2 and 3. The fields are grouped in areas of the sky that allow us to enlarge on previous surveys, test various hypotheses concerning the distribution of low mass objects in the galaxy and closely follow the planned SuperCOSMOS survey digitisation programme. To avoid image crowding and blending problems, all chosen fields avoid the Galactic plane, defined as $-20^{\circ}<b_{\mathrm{Gal}}<20^{\circ}$.

The project itself is one application of a more ambitious plan to fully digitise the $\mathrm{B}, \mathrm{R}$ and I surveys of the southern sky, to photometrically calibrate these SuperCOSMOS data, providing photographic magnitudes accurate to around 0.1 to 0.2 magnitudes and to measure proper motions where possible with a positional accuracy of a few microns and a baseline of around 10 years. 


\section{The end of the main sequence}

\subsection{Theoretical guides.}

Our knowledge of the lowest mass stars is limited to a half-dozen or so numerical models and handful of observed candidates, with large uncertainties in mass and temperature. A consistent feature of low mass models is of a minimum hydrogen-burning mass with a value of around $0.08 \mathrm{M}_{\odot}$, for solar metallicity, rising towards $0.1 \mathrm{M}_{\odot}$ for decreasing metallicities. If, during an episode of star formation, a fragment is formed which leads to an object with a mass below this value, the object's core will become degenerate before it has reached a temperature high enough to support stable hydrogen burning. Such objects are called brown dwarfs. They are capable of shining with the energy of further gravitational contraction plus brief intervals of $\mathrm{H}, \mathrm{D}$ and $\mathrm{Li}$ fusion, gradually cooling to below $10^{-5} \mathrm{~L}_{\odot}$ over $\sim 10^{9}$ years. Such objects, if they exist, occupy a whole order of magnitude in the Galactic mass function and may be the most common objects in the Galaxy. The conversion of theoretical $(\mathrm{L}, \mathrm{T})$ parameters into observational quantities (magnitude, colour) is hampered by the lack of any suitable atmospheric models, even for late dM stars. Many of the most recent models, calculated for a variety of convection and opacity parameters, consistently predict effective temperatures between $\sim 2800 \mathrm{~K}$ to $\sim 1200 \mathrm{~K}$ for objects in the range $0.08 \mathrm{M}_{\odot}$ to $0.02 \mathrm{M}_{\odot}$. Such objects have a peak flux in the near-infrared at a few microns, and contribute less than 10 per cent of their total flux to the visible $R$ and $I$ bands.

Despite many visible and infrared surveys, the properties of the very latesttype stars, beyond $\sim \mathrm{dM} 6$, are still poorly understood. Infrared technology has not reached the stage where large scale faint JHK surveys are possible and previous visible surveys have not covered a wide enough area of sky to achieve a suitable survey volume. Given a large enough areal coverage, the $\mathbf{R}$ and $\mathbf{I}$ band surveys of Schmidt telescopes provide us with an oppportunity to greatly improve our knowledge of this Galactic population.

\subsection{Observational guides}

Since there are no reliable model atmospheres for low mass stars and brown dwarfs, our colour index limits are based upon recent observations. From a study of over 300 red dwarfs (Leggett 1992), disk stars are observed with R-I colours of up to 2.3, $M_{I} \sim 13$ and $M_{R} \sim 15$. The sequence is extended further by the addition of the very red objects discussed in the survey of Hawkins \& Bessell (HB 1988). These observations show a continuation of Leggett's main sequence to $\mathrm{R}-\mathrm{I} \sim 2.5$ with $\mathrm{M}_{\mathrm{I}} \sim 15$. Henry \& McCarthy (1993) have studied a sample of dwarf binary systems within 10 parsecs using interferometry and have obtained a mass-luminosity relation which predicts a value of $M_{\mathrm{J}} \sim 11$ for objects close to $0.08 \mathrm{M}_{\odot}$. Synthetic spectra are extracted from some of these systems (Kirkpatrick \& McCarthy 1994) which estimate $\mathrm{R}-\mathrm{I} \approx 2.7$ for $\mathrm{M}_{\mathrm{J}} \approx$ 11 and $\mathrm{M}_{\mathrm{I}} \approx 15$, in agreement with HB's field candidates. Given the magnitude limits of Schmidt survey plates, these values suggest that very late $M$ dwarfs and candidate brown dwarfs can be detected at distances of over 100 parsecs, although in practice, samples within 1.5 to 2 magnitudes of the plate limit are found to be incomplete. 


\subsection{Predictions from luminosity functions.}

The area surveyed by each Schmidt field is limited by de-sensitised areas of older plates and by the vignetting of the plate which reduces the sensitivity beyond $3^{\circ}$ of the field centre. A typical measurement provides a useful area of around 27 square degrees. Recent determinations of the low mass stellar luminosity function (LF) provide an estimate of the sub-stellar space density. Differences in Henry and McCarthy's 5 and 10 parsec binary star LF suggest that there may be up to 70 undiscovered systems within 10 parsecs of the sun, some of which may contain sub-stellar companions. By integrating this implied space density over the volume covered by a typical plate, each field could contain up to 14 new late-type stars, some of which may have sub-stellar companions. How the binary LF is related to the field star LF is unknown, and since a Schmidt plate survey is limited to studying field stars, the LFs obtained by Hawkins and Bessell (HB) and by Tinney et al. (1992) provide more significant data. Extrapolating the space density of the HB survey from $M_{R}=17$ to $M_{R}=19$ gives sufficient objects to provide a single detection at $M_{R}=18$ for every 5 fields studied and one per 25 fields at $M_{R}=19$. Within the uncertainties introduced in converting between the different systems used, the bolometric LF of Tinney et al. appears to be in agreement with HB except for the faintest bins, where the HB LF appears to rise.

Variations in the local LF may be due to poor statistics but there is tentative evidence that genuinely different results could be obtained from LF determinations carried out in the northern and southern hemispheres. The HB survey suggests a possibility that their faintest objects may have a scale height as low as 150 parsecs and results from a CTI ${ }^{1}$ survey of $\mathrm{M}$ dwarfs, discussed by Kirkpatrick (1994) adds support to the idea that due to our position above the Galactic plane, a LF which doesn't take into account the true scale height of the population will give spurious results varying with the area of sky studied. To investigate this idea further we have selected three areas to comprise the initial part of the whole-sky survey. A high Galactic latitude area close to the South Galactic Pole, an area studied by Hawkins and Bessell and a mid-latitude area in the vicinity of the other two fields studied in the HB survey have been chosen. These areas coincide with the initial SuperCOSMOS survey digitisation programme which is planned to begin later this year. Some COSMOS data have already been acquired in several of the chosen fields and examples of preliminary data are used in section 3. A third area, north of the Galactic plane, in the equatorial survey region $\left(0^{\circ}>\delta>-20^{\circ}\right)$ has also been chosen. It is hoped that these areas, containing around $60 \mathrm{Schmidt}$ survey fields, will provide a definitive guide to the low mass LF and the observational properties of objects straddling the end of the main sequence.

${ }^{1}$ CCD Transit Instrument at Kitt Peak. 


\section{Calibrating COSMOS/SuperCOSMOS data}

\subsection{Photometry}

For the planned database the SuperCOSMOS intensity scale can be calibrated to the appropriate photometric system with the HST Guide star photometric catalogue (GSPC). The current GSPC consists of sequences of $\sim 6$ stars close to the centre of each Schmidt survey field. $B$ and $V$ magnitudes are provided to a limit of $\mathrm{V} \sim 16$. This is inadequate but the faint star catalogue will supplement each field with further BVR measurements which should be deep enough to allow calibration of each field to the plate limit in each of these bands. Until the publication of this information, it is neccessary to obtain photometric measurements privately and/or use previously published material.

As mentioned in section 2, calibration of individual plates shows that the number-magnitude counts peak around 1 to 2 magnitudes above the plate limit. The depth and hence completeness varies from plate to plate and so samples are limited by the poorest plate in the data set. Colour terms between photometric systems can lead to large errors in calibration. The natural systems of the UK Schmidt sky survey have well-determined colour corrections which allow for calibrations of the COSMOS measurements to an accuracy of around 0.1 to 0.2 magnitudes, depending on plate quality. Of particular importance is the difference between the $R$ and ' $O R$ ' band plates, which increases rapidly for stars redder than $R-I>1.0$. The OR band has a closer match to the Cousins system than the older $\mathrm{R}$ band, which requires separate corrections for red giants and dwarfs (Bessell 1986). For this reason, we use OR plates where possible, although $\mathrm{R}$ band plates are usually required to provide a suitable time-baseline for proper motion measurements. Having calibrated each plate in a given field, the measurements are paired, image-for-image, removing most of the spurious images found on a COSMOS data file, to give a catalogue of positions and BRI magnitudes for each image.

\subsection{Astrometry}

The pairing process used to produce the photometric catalogue is repeated for proper motion measurements. The co-ordinate transforms obtained are used to define a mean reference frame, to which each plate is then compared. To eliminate systematic differences across a plate, the master frame is divided into smaller regions and local measurements are made. The rms plate-to-plate positional accuracy is $\sim 2.4$ microns (Hawkins 1986) which gives proper motions accurate to $\sim 30$ milliarcseconds per year for a ten year baseline, typical in this survey. Further details of the technique are discussed by Hawkins (1986).

Proper motion measurements add an extra dimension to our survey, allowing us to select stars with unusual, although not neccessarily large motions. For a typical sample of late $M$ dwarfs detected with COSMOS data the majority are found to have motions that are small and close to the scatter introduced by the co-ordinate tranformations. Any outstanding objects are almost certainly very nearby $\mathrm{dM}$ stars or subdwarfs. By combining both photometric and astrometric data a reduced proper motion $\left(\mathrm{H}_{R}\right)$ diagram can be produced, which contains information about the tangential velocity of local stars as a function of apparent magnitude, thus providing a simple distance-magnitude relation. Plotting $\mathrm{H}_{R}$ 
against colour shows that the majority of these cool stars lie in a group with a gradient that follows the $\mathrm{M}_{R}$ vs $\mathrm{R}-\mathrm{I}$ relation. The outstanding objects in such a plot appear to have a lower proper motion for their colour than the sample as a whole. Such a plot appears in Bessell (1991) for the I-K colour. An interpretation is that the latest dwarfs have a lower velocity dispersion, implying a younger age than the population at higher masses, and that such objects may still be in a stage of pre-main sequence contraction. A more contentious idea is that we are seeing the young, bright sub-set of a brown dwarf population whose spatial distrubition, as inferred from visible-band surveys, may be underestimated due to older members cooling into the infrared. With the huge areal coverage of Schmidt plates and the measuring power of SuperCOSMOS, we can bring statistically useful information to this under-studied population, and help to resolve some of the uncertainities concerning the physical structure and space distribution of the lowest mass stars and brown dwarfs.

\section{Acknowledgements}

I would like to thank the Local organising commitee for subsidising my attendance and also Sandra Harrison for making the arrangements.

\section{References}

Bessell M. S., 1986, PASP, 98, 1303

Bessell M. S., 1991, AJ, 101, 662

Hawkins M. R. S. \& Bessell M. S., 1988, MNRAS, 234, 177

Hawkins M. R. S., 1986, MNRAS, 223, 845

Henry T.\& McCarthy D., 1993, AJ, 106, 773

Kirkpatrick D., 1994, in Proc. of the 8th Cambridge Workshop on Cool Stars, Stellar Systems and the Sun, in press

Kirkpatrick D. \& McCarthy D., 1994, AJ, 107, 333

Leggett S. K., 1992, ApJS, 82, 351

Tinney C., Mould J. \& Reid, I. N., 1992, ApJ, 396, 173 